\title{
Tratamiento con inyección percutánea con etanol (PEIT) de nódulos tiroideos sólidos benignos. Eficacia y experiencia en México
}

\section{Treatment of benign solid thyroid tumors with percutaneous ethanol inyection (PEIT). Eficacy and experience in Mexico}

\author{
Sigfrido Miracle-López ${ }^{1 *}$, ERnesto Rodríguez-Ayala² y CÉsAR A. SÁnchez Alanís ${ }^{3}$ \\ ${ }^{1}$ Coordinación de Endocrinología, Hospital Ángeles de las Lomas; ${ }^{2}$ Facultad de Ciencias de la Salud, Universidad Anahuac Norte; \\ ${ }^{3}$ Departamento de Radiología, Hospital Ángeles de las Lomas. Ciudad de México, México
}

\section{RESUMEN}

\begin{abstract}
En México el único tratamiento disponible abiertamente para los nódulos tiroideos fríos benignos es la cirugía, ya que solo un centro se encuentra realizando ablación con rayo láser. $\mathrm{A}$ nuestro mejor conocimiento solo existe un reporte de uso de tratamiento con inyección percutánea con etanol (PEIT) en reducción de volumen nodular en Brasil y no existen reportes en población mexicana. Presentamos nuestros primeros 15 procedimientos de PEIT en nódulos tiroideos sólidos benignos. Todos los pacientes tenían diagnóstico citopatológico de clase II de la clasificación de Bethesda. EI PEIT fue hecho bajo guía ultrasonográfica y se utilizó etanol al $99.57 \%$ en todos los procedimientos. El volumen total inyectado se calculó a un $\mathrm{cm}^{3}$ por $\mathrm{cm}^{3}$ de volumen del nódulo, dividido entre tres a cinco sesiones. Se logró reducción mayor del 50\% del volumen nodular en el $86 \%$ de los sujetos. Todos los sujetos reportaron dolor leve a moderado que duró de 36 a 48 horas, pero no impidió que los pacientes terminaran con los procedimientos. Nuestro primer reporte muestra que el PEIT consigue una reducción exitosa del volumen nodular. Consideramos que el PEIT es una segura e infrautilizada alternativa a la cirugía en individuos con adenomas tiroideos sólidos fríos benignos.
\end{abstract}

Palabras clave: Nódulos. Benignos. Ablación. Etanol. PEIT.

\section{ABSTRACT}

In Mexico, the only treatment openly available for cold benign thyroid nodules is surgery, since only one center is performing laser ablation. To our best knowledge there is only one report of the use of treatment with percutaneous ethanol injection (PEIT) in nodular volume reduction in Brazil and there are no reports in the Mexican population. We present our first fifteen PEIT procedures in benign solid thyroid nodules. All patients had a class II cytopathological diagnosis of the Bethesda classification. The PEIT was done under ultrasound guidance and $99.57 \%$ ethanol was used in all procedures. The total volume injected was calculated at one $\mathrm{cm}^{3}$ per $\mathrm{cm}^{3}$ of volume of the nodule, divided between three to five sessions. A greater than $50 \%$ reduction in nodular volume was achieved in $86 \%$ of the subjects. All subjects reported mild to moderate pain that lasted 36 to 48 hours but did not prevent patients from completing procedures. Our first report shows that PEIT achieves a successful reduction of nodular volume. We believe that PEIT is a safe and underutilized alternative to surgery in individuals with benign cold solid thyroid adenomas.

Key words: Nodules. Benign. Ablation. Ethanol. PEIT.
Correspondencia:

*Sigfrido Miracle-López

E-mail: smiracle911@msn.com
Fecha de recepción: 07-03-2020

Fecha de aceptación: 24-04-2020

DOI: 10.24875/RME.20000015
Disponible en internet: 20-07-2020

Rev Mex Endocrinol Metab Nutr. 2020;7:113-21

2462-4144 / @ 2020 Sociedad Mexicana de Nutricion y Endocrinologia, AC. Publicado por Permanyer. Éste es un artículo open access bajo la licencia CC BY-NC-ND (http://creativecommons.org/licenses/by-nc-nd/4.0/). 
Tabla 1. Estudios iniciales y resultados de la ablación térmica de nódulos tiroideos

\begin{tabular}{|c|c|c|c|c|c|c|c|}
\hline Autor & Año & País & Intervención & \# Pacientes & $\begin{array}{l}\text { Volumen } \\
\text { inicial ml }\end{array}$ & $\begin{array}{c}\text { Volumen } \\
\text { final ml }\end{array}$ & $\begin{array}{l}\text { \% de } \\
\text { reducción }\end{array}$ \\
\hline Dossing $^{18}$ & 2002 & Dinamarca & Láser & 16 & 10 & 5.4 & 46 \\
\hline Dossing $^{19}$ & 2005 & Dinamarca & Láser & 15 & 9 & 5.3 & 41.1 \\
\hline Dossing 20 & 2006 & Dinamarca & Láser & 15 & 10.1 & 5.7 & 43.6 \\
\hline Dossing $^{21}$ & 2006 & Dinamarca & Láser & 10 & 10.6 & 6.5 & 38.7 \\
\hline Gambelunche $^{22}$ & 2006 & Italia & Láser & 13 & 10.4 & 5.7 & 45.2 \\
\hline Dossing $^{23}$ & 2007 & Dinamarca & Láser & 14 & 10.6 & 4.6 & 56.6 \\
\hline Papini24 & 2007 & Italia & Láser & 21 & 11.7 & 6.2 & 47 \\
\hline Pool láser & & & & 104 & & & 45.30 \\
\hline Baek $^{25}$ & 2010 & Corea & RFA & 15 & 7.5 & 1.3 & 82.7 \\
\hline Huh $^{26}$ & 2012 & Corea & RFA & 15 & 13.3 & 3.8 & 71.4 \\
\hline$\overline{\text { Faggiano }^{27}}$ & 2012 & Italia & RFA & 20 & 13.3 & 3.2 & 75.9 \\
\hline Pool RFA & & & & 50 & & & 75.66 \\
\hline
\end{tabular}

RFA: ablación por radiofrecuencia.

\section{INTRODUCCIÓN}

Los nódulos tiroideos son masas sólidas o llenas de fluido que se forman dentro del tejido tiroideo. Los nódulos tiroideos benignos producen problemas:

- Cuando son hiperfuncionantes, porque producen hipertiroidismo ${ }^{1,2}$,

- Cuando son grandes, ya que pueden producir síntomas compresivos sobre tráquea y esófago, y pueden ocasionar alteraciones cosméticas.

La nodularidad en el tejido tiroideo es extremadamente común. En el estudio de Framigham³ ${ }^{3}$ los nódulos tiroideos clínicamente aparentes estaban presentes en el $6.4 \%$ de las mujeres y el $1.5 \%$ de los hombres. La eficacia de la terapia de supresión hormonal de hormona estimulante de la tiroides, o tirotropina (TSH) en pacientes eutiroideos con nódulos tiroideos solitarios benignos o bocio multinodular no tóxico esporádico es controvertida. La mayoría de los estudios ha demostrado que solo una minoría de los nódulos tiroideos disminuyen su tamaño en los pacientes que toman terapia de supresión con hormonas tiroideas ${ }^{4,5}$, y la Asociación Americana de Tiroides en sus guías ${ }^{6}$ refiere que la supresión sistemática de la TSH con levotiroxina de los nódulos tiroideos benignos en áreas suficientes de yodo no está recomendada. El tratamiento de los nódulos tiroideos sólidos con ablación térmica ${ }^{7}$, tanto por ablación por radiofrecuencia (RFA) como con ablación por rayo láser ha sido muy exitoso ${ }^{7-14}$, e incluso se ha llevado a cabo un metaanálisis comparando el uno con el otro y el costo-eficacia de la RFA ya ha sido probado frente a la cirugía ${ }^{15-17}$. En México el único tratamiento disponible abiertamente para los nódulos tiroideos fríos es la cirugía, ya que una sola persona en México está realizando rayo láser. Aunque el tratamiento de los nódulos tiroideos sólidos con RFA y con rayo láser ha sido muy exitoso, esta tecnología no está disponible abiertamente en nuestro país, y sus costos pueden ser muy elevados. En la tabla 1 se muestran los estudios iniciales del uso de ablación por medios térmicos de los nódulos tiroideos sólidos benignos. Esta limitante en la disponibilidad de tratamientos no invasivos pone una enorme demanda sobre los recursos de salud del Estado, considerando que no todos los Estados de la república cuentan con un cirujano experto que pueda realizar el tratamiento quirúrgico de estos nódulos. La finalidad del trabajo aquí presentado es demostrar que la ablación percutánea con etanol es una opción terapéutica eficaz y efectiva, para el tratamiento de los pacientes con nódulos tiroideos sólidos, benignos, hipofuncionantes. Estudios más recientes ${ }^{28,29}$ han cuestionado la eficacia del tratamiento con inyección percutánea con etanol (PEIT) para tratar nódulos sólidos. El mecanismo 
Tabla 2. Clasificación de Bethesda ${ }^{33}$

\begin{tabular}{llcl}
\hline Categoría diagnóstica & Riesgo de malignidad & Paso siguiente \\
\hline I & No diagnóstica o insatisfactoria & $5-10 \%$ & Repetir la biopsia guiada por ultrasonido \\
\hline II & Benigna & $0-3 \%$ & Seguimiento clínico y ultranográfico \\
\hline III & Atipia de significado incierto & $10-30 \%$ & Repetir la biopsia, marcadores moleculares o lobectomía \\
\hline IV & Neoplasia folicular & $25-40 \%$ & Marcadores moleculares o lobectomía \\
\hline V & Sospechosa de malignidad & $50-75 \%$ & Tiroidectomía casi total o lobectomía \\
\hline VI & Maligna & $97-99 \%$ & Tiroidectomía casi total o lobectomía \\
\hline
\end{tabular}

terapéutico del PEIT funciona aplicando etanol de muy alta pureza (95-99\%). El etanol tiene varias uniones de hidrogeno en una sola molécula y se disuelve rápidamente al contacto con el agua. Se mecanismo de acción es una combinación de 2 efectos: 1) necrosis coagulativa de los tejidos por deshidratación celular y desnaturalización de las proteínas debida a la difusión, y 2) necrosis isquémica por formación de trombos en pequeños vasos sanguíneos en los tejidos periaplicación, lo que lleva a una fibrosis y atrofia del nódulo. Se piensa que el mecanismo principal es necrosis coagulativa para los nódulos quísticos y necrosis isquémica para los nódulos sólidos ${ }^{30}$. A nuestro mejor conocimiento, los únicos estudios reportados en pacientes latinoamericanos de tratamiento para la normalización de las pruebas de función tiroidea de nódulos tiroideos hiperfuncionantes con PEIT es el estudio publicado en 2015 de nuestra autoría ${ }^{31}$ y por el equipo de Camila Luhm Silva Pérez en Brasil en $2016^{32}$, el cual incluye datos de reducción del volumen nodular, y no existen estudios publicados en México acerca de la reducción en tamaño de nódulos tiroideos sólidos.

\section{MATERIAL Y MÉTODOS}

\section{Universo del estudio}

Todos los pacientes mexicanos que acuden a la consulta del investigador principal, que son mayores de 18 años con uno o más nódulos tiroideos sólidos (definidos como tumores puramente sólidos o predominantemente sólidos con menos del $10 \%$ de componente quístico) que ocasionen sintomatología o sean cosméticamente indeseables, y que tengan un diagnóstico previo de Bethesda II (Tabla 2) por biopsia por aspiración con aguja fina.

\section{Descripción del estudio}

Se recogió información en un hospital del Estado de México, el Hospital Ángeles Lomas (privado), del 1 de mayo del 2017 al 1 de mayo del 2019. El PEIT se realizó bajo guía ultrasonográfica (MINDRAY $5^{\mathrm{TM}}$ ). Se utilizó alcohol etílico desnaturalizado 40B al 99.91\% (JT Baker) en todos los procedimientos. EI volumen total inyectado se calculó a $1 \mathrm{~cm}^{3}$ de etanol por $\mathrm{cm}^{3}$ de volumen nodular, dividido entre tres a cinco sesiones, con un volumen máximo de etanol aplicado en un solo procedimiento de $5 \mathrm{~cm}^{3}$ :

1) Menos de $5 \mathrm{~cm}^{3}$ : tres sesiones.

2) Entre 5 y $10 \mathrm{~cm}^{3}$ : cuatro sesiones.

3) Más de $10 \mathrm{~cm}^{3}$ : cinco sesiones.

Las sesiones se aplicaron con una semana de separación entre cada una.

Todos los ultrasonidos fueron realizados por un mismo medico radiólogo, quien fue el encargado de determinar el volumen nodular inicial, intermedio y final. Todos los pacientes contaban con un estudio citopatológico del nódulo tiroideo que tratar, el análisis citopatológico fue realizado por un equipo constituido por dos médicos citopatólogos, requiriendo de concordancia entre los dos para poder incluir a los pacientes ${ }^{33}$

Criterios de inclusión:

- Pacientes mexicanos de sexo indistinto mayores de 18 años. 
- Pacientes con nódulos tiroideos benignos (definido como clase funcional Bethesda II) que causen problemas cosméticos $\mathrm{y} / \mathrm{o}$ sintomáticos.

- Pacientes que acudan a la consulta del investigador principal.

- Criterios de exclusión:

- Pacientes con nódulos tiroideos categoría de Bethesda III o superior.

- Pacientes con nódulos tiroideos benignos que no causen problemas cosméticos y/o sintomáticos.

- Pacientes que no puedan cumplir con el seguimiento ultrasonográfico (al mes, 3 meses y 6 meses).

- Pacientes que no acudan a la consulta del investigador principal.

Criterios de eliminación:

- Pacientes que no terminen con las sesiones de tratamiento establecidas.

- Pacientes que decidan retirarse del estudio.

\section{Valoración inicial del paciente y los nódulos}

Todos los pacientes contaron al inicio del estudio con:

- Un diagnóstico histocitológico de benignidad (Bethesda II) del nódulo que tratar.

- Características ultrasonográficas del nódulo y del cuello.

- Un cálculo de volumen nodular.

- Exámenes de laboratorio:

- Tiempos de coagulación.

- Pruebas de función tiroidea.

\section{Estandarización de medidas ultrasonográficas}

Los ultrasonidos fueron realizados en exclusividad por un médico radiólogo; el procedimiento de ablación fue realizado en exclusividad por el investigador principal, con la guía ultrasonográfica del radiólogo antes mencionado; las mediciones del volumen nodular estuvieron a cargo exclusivamente del médico radiólogo. Para determinar el volumen nodular se utilizó la ecuación:

$\mathrm{V}=\pi \mathrm{abc} / 6$

en donde $V$ es el volumen, a es el diámetro máximo, y b y c los otros dos diámetros perpendiculares.

Se consideró éxito del tratamiento una reducción del volumen a los 6 meses $>50 \%$.

\section{Análisis estadístico}

Se realizó un análisis descriptivo en el cual se expresan los datos en porcentaje en la media y desviación estándar. Se consideró diferencia significativa cuando $p<0.05$. El estudio busca demostrar la no inferioridad $^{34}$ del PEIT frente a la RFA y la ablación por rayo láser, por lo que se calculó la varianza, las medidas de tendencia central y de dispersión para las diversas variables, las variables numéricas continuas entre grupos se analizaron mediante un análisis de la varianza (ANOVA) de Fisher, ya que se trata de variables cuantitativas con muestras relacionadas de más de dos grupos. La diferencia significativa en la reducción de volumen se realizó mediante prueba $t$ pareada bilateral tomando en consideración que tenemos variables cuantitativas relacionadas de distribución normal. Para validar el análisis de la precisión en las mediciones de los nódulos se realizó un análisis de varianza y el cálculo del coeficiente de correlación intraclase ICC2 C1 para evaluar la consistencia de las mediciones. Para conocer la posible influencia del observador en el error de medición se calculó el coeficiente de correlación intraclase ICC2 A1, que cuantifica el absoluto acuerdo:

$\mathrm{CCl}={ }_{P}^{* 2}{ }_{P}^{* 2}{ }_{P}+{ }^{* 2}{ }_{O}+{ }^{* 2}{ }_{R}$

\section{Consideraciones éticas}

El estudio se apega estrictamente a lo estipulado en la Ley General de Salud en Materia de Investigación, lo concerniente a aspectos éticos de la investigación en seres humanos y de la bioseguridad de las investigaciones (Título Segundo Capítulo I y 
Título IV, Capítulo I). Así mismo, observa en lo pertinente lo establecido por la Norma Oficial NOM087-ECOL-SSA 1-2002, protección ambiental-salud ambiental- residuos peligrosos biológico-infecciosos. Clasificación y especificaciones de manejo (NOM-087); concretamente, los artículos XIV (fracción $\mathrm{V}$ y VI), XVI y XVII y las metas 1, 2 y 3 de lo estipulado por la Joint Comission para seguridad del participante.

\section{Monitoreo de la evolución de los participantes después de la ablación}

a) El investigador principal proporcionó los números telefónicos u otros medios mediante los cuales la persona o un familiar pudiese comunicarse si surgiera algún problema relacionado con el sitio de punción e inyección de etanol (dolor, sangrado, etc.).

b) El investigador principal se comunicó de forma sistemática con él o la participante (aun sin que lo hubieran solicitado) a las $24 \mathrm{~h}$ del evento para saber de su evolución.

El proyecto de investigación contó con la aprobación del Comité de Ética Local y del Comité de Ética e Investigación de la facultades de Bioética y de Ciencias de la Salud de la Universidad Anahuac Campus México Norte.

\section{RESULTADOS}

Ingresaron al estudio un total de 15 pacientes, 11 mujeres (73\%) y 4 hombres (27\%), con una edad media de $45.93 \pm 13.25$ años, con un rango entre 20 y 73 años. Todos los pacientes cumplieron los criterios de inclusión, con diagnóstico de Bethesda II, nueve con nódulo folicular benigno (60\%), cuatro con nódulo adenomatoso (27\%) y dos con bocio multinodular no tóxico (13\%) (Tablas 3 y 4). El volumen medio de los nódulos fue de $14.46 \pm 19 \mathrm{~cm}^{3}$, con un rango de 0.809 a $73.47 \mathrm{~cm}^{3}$; un volumen medio final de $5.24 \pm 8.44 \mathrm{~cm}^{3}$, con un rango de 0.31 a $34.11 \mathrm{~cm}^{3}$; el porcentaje de reducción medio
Tabla 3. Demografía de los pacientes

\begin{tabular}{lllll}
\hline Paciente & Edad & Sexo & Raza & $\begin{array}{c}\text { Enfermedades } \\
\text { tiroideas } \\
\text { concomitantes }\end{array}$ \\
\hline 1 & 47 & $F$ & Latina & No \\
\hline 2 & 42 & $F$ & Latina & No \\
\hline 3 & 44 & $F$ & Latina & No \\
\hline 4 & 43 & $F$ & Latina & No \\
\hline 5 & 20 & $F$ & Latina & No \\
\hline 7 & 57 & M & Latina & No \\
\hline 8 & 36 & M & Latina & No \\
\hline 9 & 46 & $F$ & Latina & No \\
\hline 10 & 43 & $F$ & Latina & No \\
\hline 11 & 46 & $F$ & Latina & No \\
\hline 12 & 54 & $M$ & Latina & No \\
\hline 13 & 64 & $F$ & Latina & Si \\
\hline 14 & 31 & $F$ & Latina & No \\
\hline 15 & 73 & $M$ & Latina & No \\
\hline
\end{tabular}

*Paciente sometido a tiroidectomía con un diagnóstico final de microcarcinoma papilar de tiroides.

F: femenino; M: masculino.

fue del $63 \pm 17 \%$, con un rango mínimo del $27 \%$ y un máximo del $89 \%(\mathrm{p}=0.0055)$. Dos pacientes (13.34\%) no completaron el estudio, uno de ellos abandonó por complicaciones después de tres sesiones y un segundo fue perdido al seguimiento (6.67\%), con un solo seguimiento de volumen al mes postratamiento. Ya que el estudio tiene intención de tratar (ITT), consideramos a estos dos paciente como falla de tratamiento. Se presentaron complicaciones en cinco pacientes (33\%), de las cuales: extravasación de etanol fuera del nódulo en dos pacientes, disfonía transitoria que requirió de uso de dexametasona $8 \mathrm{mg}$ en un paciente, hematoma autolimitado en dos pacientes y tiroiditis transitoria en dos pacientes. Trece de los 15 pacientes $(86.67 \%)$ lograron una reducción del volumen nodular de más del $50 \%$ a los seis meses después de la última aplicación de etanol (Tabla 5). Para evaluar la consistencia de las mediciones se utilizó el cálculo del coeficiente de correlación intraclase ICC2 y para conocer la posible influencia del observador en el error de medición se calculó el coeficiente de correlación intraclase ICC2 A1, que cuantifica el absoluto acuerdo, siendo los resultados $0.80 \mathrm{y}$ 
Tabla 4. Características de los nódulos

\begin{tabular}{lccll}
\hline Paciente & Volumen inicial & Composición & Funcionalidad & BAAF \\
\hline 1 & 10.89 & Sólido & Hipofuncional & Nódulo folicular benigno \\
\hline 2 & 1.1 & Sólido & Hipofuncional & Bocio multinodular \\
\hline 3 & 0.89 & Sólido & Hipofuncional & Nódulo adenomatoso \\
\hline 5 & 8.23 & Sólido & Hipofuncional & Nódulo folicular benigno \\
\hline 6 & 19 & Sólido & Hipofuncional & Nódulo folicular benigno \\
\hline 7 & 1.43 & Sólido & Hipofuncional & Nódulo folicular benigno \\
\hline 8 & 5.2 & Sólido & Hipofuncional & Nódulo folicular benigno \\
\hline 9 & 7.98 & Sólido & Hipofuncional & Nódulo dominante de bocio \\
\hline 10 & 2.6 & Sólido & Hipofuncional & Nódulo adenomatoso \\
\hline 11 & 26.57 & Sólido & Hipofuncional & Bocio coloide \\
\hline 13 & 73.47 & Sólido & Hipofuncional & Nódulo folicular benigno \\
\hline 14 & 5.86 & Sólido & Hipofuncional & Nódulo folicular benigno \\
\hline 15 & 11.2 & Sólido & Hipofuncional & Nódulo folicular benigno \\
\hline
\end{tabular}

BAAF: biopsia por aspiración con aguja fina.

Tabla 5. Resultados del tratamiento

\begin{tabular}{|c|c|c|c|c|c|c|}
\hline Paciente & $\begin{array}{l}\text { Volumen inicial } \\
\qquad\left(\mathrm{en} \mathrm{cm}^{3}\right)\end{array}$ & $\begin{array}{l}\text { Volumen final } \\
\qquad\left(\mathrm{en}^{3} \mathrm{~cm}^{3}\right)\end{array}$ & $\begin{array}{l}\text { Número de } \\
\text { sesiones }\end{array}$ & $\begin{array}{l}\text { Porcentaje de } \\
\text { reducción }\end{array}$ & Éxito & Complicaciones* \\
\hline
\end{tabular}

\begin{tabular}{|c|c|c|c|c|c|c|}
\hline 1 & 10.89 & 2.1 & 4 & 81 & $S$ & 1 \\
\hline 2 & 1.1 & 0.481 & 3 & 56 & $S$ & $\mathrm{~N}$ \\
\hline 3 & 0.89 & 0.33 & 3 & 59 & $S$ & $\mathrm{~N}$ \\
\hline 4 & 8.23 & 2.01 & 4 & 76 & $S$ & $\mathrm{~N}$ \\
\hline 5 & 19 & 7.09 & 5 & 63 & $S$ & $\mathrm{~N}$ \\
\hline 6 & 1.43 & 0.48 & 3 & 66 & $S$ & $\mathrm{~N}$ \\
\hline 7 & 5.2 & 1.47 & 3 & 72 & $S$ & $\mathrm{~N}$ \\
\hline 8 & 7.98 & 3.55 & 4 & 56 & $S$ & 3,4 \\
\hline 9 & 2.6 & 0.31 & 3 & 88 & $S$ & $\mathrm{~N}$ \\
\hline 10 & 26.57 & 2.935 & 5 & 89 & $S$ & 3,4 \\
\hline 11 & 73.47 & 34.11 & 5 & 54 & $S$ & $\mathrm{~N}$ \\
\hline $12^{+}$ & 5.86 & 5.06 & $3^{*}$ & 14 & $\mathrm{~N}$ & 1 \\
\hline $13^{\ddagger}$ & 11.2 & 6.89 & $5^{*}$ & 39 & $\mathrm{~N}$ & 2 \\
\hline 14 & 25.16 & 9.42 & 5 & 63 & $S$ & $\mathrm{~N}$ \\
\hline 15 & 13.77 & 3.23 & 5 & 77 & $S$ & $N$ \\
\hline
\end{tabular}

*1: extravasasión de etanol fuera del nódulo; 2: disfonía transitoria; 3: hematoma autolimitado; 4: tiroiditis transitoria.

tPerdido al seguimiento, última medición del nódulo al mes postratamiento.

‡Discontinúo el tratamiento después de la tercera aplicación por complicaciones.

0.82 respectivamente. El ANOVA de Fisher demostró que no hubo diferencia significativa entre el PEIT y la RFA en la tasa de éxito de reducción del volumen nodular (Fig. 1), demostrándose una tendencia de superioridad entre el PEIT y el rayo láser, y la RFA y el rayo láser (Tabla 6).

\section{DISCUSIÓN}

Basándonos en datos poblacionales reportados previamente ${ }^{35-41}$, podríamos estimar que en México alrededor de 63 millones de personas serían portadores 


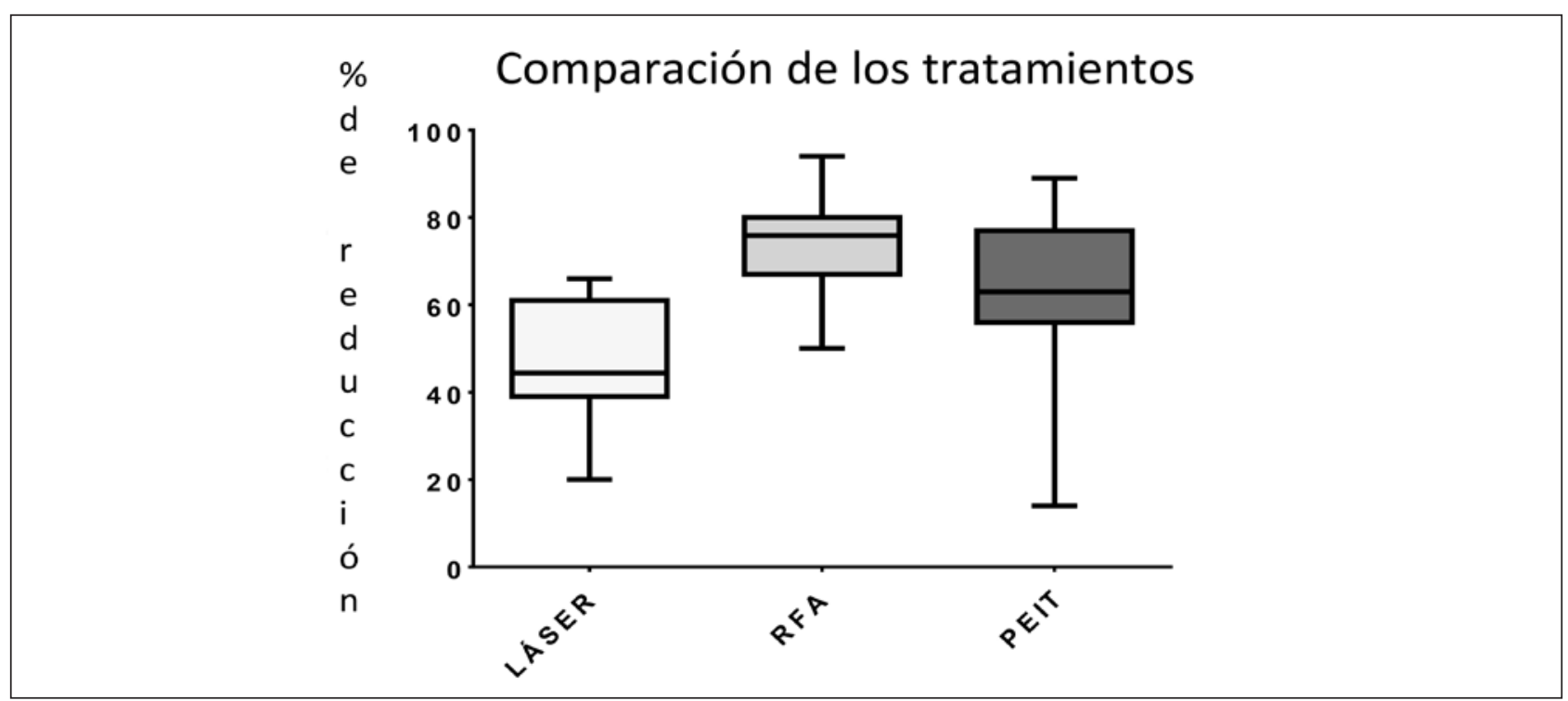

Figura 1. Comparación de la reducción del volumen nodular con láser, ablación por radiofrecuencia (RFA) e inyección percutánea con etanol (PEIT).

Tabla 6. Análisis de ANOVA comparando los tres tratamientos no quirúrgicos de ablación de nódulos tiroideos sólidos: láser, ablación por radiofrecuencia e inyección percutánea con etanol

\begin{tabular}{|c|c|c|c|c|c|c|c|}
\hline Número de familias & 1 & & & & & & \\
\hline $\begin{array}{l}\text { Número de comparativos } \\
\text { por familia }\end{array}$ & 3 & & & & & & \\
\hline Alfa & 0.05 & & & & & & \\
\hline $\begin{array}{l}\text { Prueba de comparativos } \\
\text { múltiples de Dunn }\end{array}$ & $\begin{array}{l}\text { Rango } \\
\text { medio } 1\end{array}$ & $\begin{array}{l}\text { Rango } \\
\text { medio } 2\end{array}$ & $\begin{array}{c}\text { Diferencia } \\
\text { media de } \\
\text { rangos }\end{array}$ & Significativo & Resumen & $\mathrm{n} 1$ & $\mathrm{n} 2$ \\
\hline Láser vs. RFA & 14.75 & 37.71 & -22.96 & $\mathrm{SI}$ & $* * * *$ & 20 & 17 \\
\hline Láser vs PEIT & 14.75 & 29.47 & -14.72 & $\mathrm{SI}$ & * & 20 & 15 \\
\hline RFA vs PEIT & 37.71 & 29.47 & 8.239 & NO & ns & 17 & 15 \\
\hline
\end{tabular}

RFA: ablación por radiofrecuencia; PEIT: ablación percutánea con etanol; ns: no significativo.

de uno o más nódulos tiroideos, de los cuales alrededor de 15 millones podrían ser portadores de uno o más nódulos mayores de $1 \mathrm{~cm}$. En México el único tratamiento disponible abiertamente para los nódulos tiroideos fríos que ocasionan síntomas compresivos o son estéticamente desagradables es la cirugía. El PEIT se ha utilizado por más de 15 años para tratar lesiones tiroideas tanto benignas como malignas. Este procedimiento puede enseñarse con relativa facilidad no solo a los especialistas en endocrinología (1,784 en el 2018), sino también a los cirujanos generales (10,982), cirujanos de cabeza y cuello, otorrinolaringólogos, cirujanos oncólogos $(3,615)$ y médicos radiólogos $(3,819)$, lo que aumentaría el número de especialista capaces de tratar esta patología de 3,615 (4.44 por cada 100,00 habitantes) a 20,200 (24.81 por cada 100,000 habitantes). El procedimiento puede realizarse fuera de los quirófanos, en un tiempo aproximado de 30 minutos y sin requerir que el paciente sea hospitalizado, reduciendo los días de incapacidad de 10 días a solo un día. El tratamiento con PEIT abre la posibilidad a que en un país con las limitaciones económicas y tecnológicas como el nuestro se pueda 
atender a todos los pacientes con nódulos tiroideos sólidos benignos que causan alteraciones cosméticas y/o sintomatológicas en el consultorio médico a un costo muy reducido y con un mínimo de días de incapacidad, lo que permitiría un acceso universal al tratamiento de estos problemas. Lo que se debió comprobar con el estudio es que el PEIT fuera no inferior como tratamiento en comparación con la RFA y el rayo láser, tomándose como éxito una reducción del $50 \%$ o más del volumen nodular inicial. Para esto primero reportamos la reducción de volumen en nuestros pacientes y la tasa de éxito del tratamiento con PEIT, tomando en cuenta la consistencia de las mediciones con el cálculo del coeficiente de correlación intraclase ICC2 y para conocer la posible influencia del observador en el error de medición se calculó el coeficiente de correlación intraclase ICC2 A1, que cuantifica el absoluto acuerdo, siendo los resultados 0.80 y 0.82 respectivamente, lo que se considera una buena correlación. Posteriormente se los comparó con los reportados en la literatura universal acerca de la RFA y del rayo láser, ya que al no ser procedimientos disponibles abiertamente en nuestro país no nos fue posible realizar una comparación propia de estos tratamientos. Para lograr demostrar la no inferioridad se utilizó un ANOVA de Fisher, ya que se trata de variables cuantitativas con muestras relacionadas de más de dos grupos. La diferencia significativa en la reducción de volumen se realizó mediante prueba t pareada bilateral, tomando en consideración que tenemos variables cuantitativas relacionadas de distribución normal. El análisis de Kruskal-Wallis demostró una no inferioridad del PEIT frente a la RFA y una tendencia a la superioridad de la RFA frente al láser y del PEIT frente al láser (el objetivo del estudio era mostrar no inferioridad, por lo que esta tendencia hacia la superioridad del PEIT frente al rayo láser tendrá que ser demostrada en futuros estudios). Nuestro porcentaje de complicaciones no es superior a lo reportado en estudios previos de ablación con PEIT, ni tampoco en lo reportado en estudios de ablación con RFA o láser. En futuros estudios realizaremos análisis de costos para valorar que el procedimiento de ablación con etanol es de costo-minimización en comparación con los otros tratamientos disponibles en el mundo.

\section{CONCLUSIONES}

Nuestro primer reporte muestra que el PEIT logra una reducción exitosa del volumen nodular. Consideramos que el PEIT es una segura e infrautilizada alternativa a la cirugía en individuos con adenomas tiroideos sólidos fríos benignos. Es necesario hacer un estudio de costo-minimización comparando las alternativas quirúrgicas y de ablación térmica de los nódulos tiroideos sólidos benignos para poder sugerir la implementación de esta forma de tratamiento en el ámbito de la medicina pública y poder justificar su entrenamiento en los programas de residencias médicas pertinentes.

FINANCIAMIENTO

Los autores no recibieron patrocinio para llevar a cabo este articulo.

\section{CONFLICTO DE INTERESES}

Los autores declaran no tener conflicto de intereses alguno.

\section{RESPONSABILIDADES ÉTICAS}

Protección de personas y animales. Los autores declaran que los procedimientos seguidos se conformaron a las normas éticas del comité de experimentación humana responsable y de acuerdo con la Asociación Médica Mundial y la Declaración de Helsinki.

Confidencialidad de los datos. Los autores declaran que han seguido los protocolos de su centro de trabajo sobre la publicación de datos de pacientes.

Derecho a la privacidad y consentimiento informado. Los autores han obtenido el consentimiento 
informado de los pacientes y/o sujetos referidos en el artículo. Este documento obra en poder del autor de correspondencia.

\section{BIBLIOGRAFÍA}

1. Frost L, Vestergaard P, Mosekilde L. Hyperthyroidism and risk of atrial fibrillation of flutter: a population-based study. Arch Intern Med. 2004;164(15):1675.

2. Ross DS. Hyperthyroidism, thyroid hormone therapy, and bone. Thyroid. 1994;4(3):319.

3. Vander JB, Gaston EA, Dawber TR. The significance of nontoxic thyroid nodules. Final report of a 15-year study of the incidence of thyroid malignancy. Ann Intern Med. 1968;69(3):537.

4. Berghout A, Wiersinga WM, Drexhage HA, Smits NJ, Touber JL . Comparison of placebo with L-thyroxine alone or with carbimazole for treatment of sporadic non-toxic goitre. Lancet. 1990;336(8709):193.

5. Wémeau JL, Caron P, Schvartz C, Schlienger JL, Orgiazzi J, Cousty C, et al. Effects of thyroid-stimulating hormone suppression with levothyroxine in reducing the volume of solitary thyroid nodules and improving extranodular nonpalpable changes: a randomized, double-blind, placebo-controlled trial by the French Thyroid Research Group. J Clin Endocrinol Metab. 2002;87(11):4928.

6. Haugen BR, Alexander EK, Bible KC, Doherty GM, Mandel SJ, Nikiforov YE, et al. 2015 American Thyroid Association Management Guidelines for Adult Patients with Thyroid Nodules and Differentiated Thyroid Cancer: The American Thyroid Association Guidelines Task Force on Thyroid Nodules and Differentiated Thyroid Cancer. Thyroid. 2016;26(1):1.

7. Mainini AP, Monaco C, Pescatori LC, De Angelis C, Sardanelli F, Sconfienza LM, et al. Image-guided thermal ablation of benign thyroid nodules J Ultrasound. 2017;20:11-22.

8. Jeong WK, Baek JH, Rhim H, Kim YS, Kwak MS, Jeong HJ, et al. Radiofrequency ablation of benign thyroid nodules: safety and imaging followup in 236 patients. Eur Radiol. 2008;18:1244-50.

9. Baek JH, Kim YS, Lee D, Huh JY, Lee JH. Benign predominantly solid thyroid nodules: prospective study of efficacy of sonographically guided radiofrequency ablation versus control condition. AJR Am J Roentgenol. 2010;194:1137-42.

10. Sung JY, Kim YS, Choi H, Lee JH, Baek JH. Optimum first-line treatment technique for benign cystic thyroid nodules: ethanol ablation or radiofrequency ablation? AJR Am J Roentgenol. 2011;196:W210-W214.

11. Baek JH, Moon WJ, Kim YS, Lee JH, Lee D. Radiofrequency ablation for the treatment of autonomously functioning thyroid nodules. World J Surg. 2009;33:1971-7.

12. Kim JH, Baek JH, Lim HK, Na DG. Summary of the 2017 thyroid radiofrequency ablation guideline and comparison with the 2012 guideline. UItrasonography. 2019;38(2):125-134. doi:10.14366/usg.18044

13. Spiezia S, Vitale G, Di Somma C, Pio Assanti A, Ciccarelli A, Lombardi G, et al. Ultrasound-guided laser thermal ablation in the treatment of autonomous hyperfunctioning thyroid nodules and compressive nontoxic nodular. Thyroid. 2003;13(10):941-7.

14. Gambelunghe G, Fede R, Bini V, Monacelli M, Avenia N, D'Ajello M, et al. Ultrasound-guided interstitial laser ablation for thyroid nodules is effective only at high total amounts of energy: results from a three-year pilot study. Surg Innov. 2013;20(4):345-50.

15. Yue WW, Wang SR, Li XL, Xu HX, Lu F, Sun LP, et al. Quality of life and cost-effectiveness of radiofrequency ablation versus open surgery for benign thyroid nodules: a retrospective cohort study. Sci Rep. 2016:6:37838.

16. Chen F, Tian G, Kong D, Zhong L, Jiang T. Radiofrequency ablation for treatment of benign thyroid nodules: A PRISMA-compliant systematic review and meta-analysis of outcome. Medicine. 2016; 95(34):e4659.

17. Ha EJ, Baek JH, Kim KW, Pyo J, Lee JH, Baek SH, et al. Comparative efficacy of radiofrequency and laser ablation for the treatment of benign thyroid nodules: Systematic review including traditional pooling and bayesian network meta-analysis. Clin Endocrinol Metab. 2015; 100(5):1903-11.
18. Døssing H, Bennedbaek FN, Karstrup S, Hegedüs L. Benign solitary solid cold thyroid nodules: US-guided interstitial laser photocoagulation-initial experience. Radiology. 2002;225:53-57.

19. Døssing $H$, Bennedbaek FN, Hegedüs L. Effect of ultrasound-guided interstitial laser photocoagulation on benign solitary solid cold thyroid nodules - a randomised study. Eur J Endocrinol. 2005;152:341-345

20. Døssing $H$, Bennedbaek FN, Hegedüs L. Beneficial effect of combined aspiration and interstitial laser therapy in patients with benign cystic thyroid nodules: a pilot study. Br J Radiol. 2006;79:943-947

21. Døssing H, Bennedbaek FN, Hegedüs L. Effect of ultrasound-guided interstitial laser photocoagulation on benign solitary solid cold thyroid nodules: one versus three treatments. Thyroid. 2006;16:763-768

22. Gambelunghe G, Fatone C, Ranchelli A, Fanelli C, Lucidi P, Cavaliere A, et al. . A randomized controlled trial to evaluate the efficacy of ultrasound-guided laser photocoagulation for treatment of benign thyroid nodules. J Endocrinol Invest. (2006) 29:RC23-6. 10.1007/BF03347368

23. Døssing H, Bennedbaek FN, Bonnema SJ, Grupe P, Hegedüs L. Randomized prospective study comparing a single radioiodine dose and a single laser therapy session in autonomously functioning thyroid nodules. Eur J Endocrinol. 2007;157:95-100

24. Papini E, Guglielmi G, Bizzarri G, Graziano F, Bianchini A, Brufani C,et al Treatment of benign cold thyroid nodules: a randomized clinical trial of percutaneous laser ablation versus levothyroxine therapy or follow-up. Thyroid. 2007 Mar; 17(3): 229-235. doi: 10.1089/thy.2006.0204

25. Baek JH, Kim YS, Lee D, Huh JY, Lee JH. Benign predominantly solid thyroid nodules: prospective study of efficacy of sonographically guided radiofrequency ablation versus control condition. AJR Am J Roentgenol. 2010;194:1137-1142

26. Huh JY, Baek JH, Choi H, Kim JK, Lee JH. Efficacy of additional treatment session of radiofrequency ablation for symptomatic benign thyroid nodules: A prospective randomized study. Radiology. 2012 Jun;263(3):90916. doi: 10.1148/radiol.12111300. Epub 2012 Mar 21.

27. Faggiano A, Ramundo V, Assanti AP, Fonderico F, Macchia PE, Misso C, et al. Thyroid nodules treated with percutaneous radiofrequency thermal ablation: a comparative study. J Clin Endocrinol Metab. 2012;97: 4439-4445

28. Baek JH, Lee JH, Valcavi R, Pacella CM, Rhim H, Na DG. Thermal ablation for benign thyroid nodules: radiofrequency and laser. Korean J Radiol. 2011:12(5):525-40.

29. Kim YJ, Baek JH, Ha EJ, Lim HK, Lee JH, Sung JY, et al. Cystic versus predominantly cystic thyroid nodules: efficacy of ethanol ablation and analysis of related factors. Eur Radiol. 2012:22(7):1573-8.

30. Hahn SY, Shin JH, Na DG, Ha EJ, Ahn HS, Lim HK, et al. Ethanol ablation of the thyroid nodules: 2018 Consensus Statement by the Korean Society of Thyroid Radiology. Korean J Radiol. 2019;20(4):606-20.

31. Miracle LS. Inyección percutánea de etanol para el tratamiento de los nódulos tiroideos sólidos hiperfuncionantes. Acta Médica Grupo Ángeles. 2015;13(4):229-31.

32. Perez CL, Fighera TM, Miasaki F, Mesa Junior CO, Paz Filho GJ, Graf H, et al. Evaluation of percutaneous etanol injections in benign thyroid nodules. Arq Bras Endocrinol Metab. 2014;58(9):912-7.

33. Cibas ES, Ali SZ. The 2017 Bethesda system for reporting thyroid cytopathology. Thyroid. 2017;27(11):1341-46

34. Ferreira-González I. Bases para la interpretación de los estudios de no inferioridad: a propósito de los estudios ROCKET-AF, RE-LY y ARISTOTLE. Rev Esp Cardiol. 2014;67(6):432-5.

35. Mortensen JD, Woolner LB, Bennet WA. Gross and microscopic findings in clinically normal thyroid glands. J Clin Endocrinol Metab. 1955;15: 1270-80.

36. Ezzat S, Sarti DA, Cain DR, Braunstein GD. Thyroid incidentalomas. Prevalence by palpation and ultrasonography. Arch Intern Med. 1994;154(16):1838.

37. Brander A, Viikinkoski P, Nickels J, Kivisaari L. Thyroid gland: US screening in a random adult population. Radiology. 1991;181(3):683.

38. Reiners C, Wegscheider K, Schicha H, Theissen P, Vaupel R, Wrbitzky R, et al. Prevalence of thyroid disorders in the working population of Germany: ultrasonography screening in 96,278 unselected employees. Thyroid. 2004;14(11):926

39. Rice CO. Incidence of nodules in the thyroid. Arch Surg. 1932;24:505.

40. Mortensen JD, Woolner LB, Bennet WA. Gross and microscopic findings in clinically normal thyroid glands. J Clin Endocrinol Metab. 1955; 15(10):1270.

41. Tan GH, Gharib H. Thyroid Incidentalomas: management approaches to non palpable nodules discovered incidentally on thyroid imaging. Ann Intern Med. 1997;126(3):226. 